\title{
Graphs as models of large-scale biochemical organization
}

\author{
Pau Fernández and Ricard V. Solé
}

March 10, 2004

\section{Introduction}

Cells have many different types of molecules interacting in space and time to produce the coordinated behavior we observe. They are a beautiful example of complex systems, which are difficult to study in their entirety due to the overwelming number of components they have. However, allowing for a certain degree of simplification, one can always start looking at these systems by simply measuring what pairs of units in these multicomponent systems engage in some form of interaction. This crude, discrete information can be a very informative first step towards an understanding of cells as an integrated whole, and provides the most simple global picture one can obtain of these systems: it's underlying network.

Networks pervade biology, and they are present at many different spatial and temporal scales, from molecular biology to large-scale evolution. By understanding the possible scenarios responsible for the emergence of their architecture, great insight can be gained on their evolutionary origins [Solé et al., 2002a]. As a first example, we can consider the case of protein molecules. On the one hand, they can be viewed simply as the units on top of which a complex system like the cell is built. But on the other hand, they count as complex systems in their own right, given the complexity of their structure and folding process. A three-dimensional structure of a linear biopolymer like a protein can, in fact, be roughly described by its contact structure, that is, by the list of all pairs of monomers that are spatial neighbours, given some cut-off distance. Figure 1A shows the structure of a portion of a protein, and figure 1B shows its contact matrix, the simplest way to quickly view the contact pairs. In this matrix, rows and columns represent each aminoacid in the sequence, and a square is drawn black when the corresponding aminoacids are in close proximity in the three dimensional structure.

Using this matrix, one can actually construct a network, shown in figure $1 \mathrm{C}$, in which the vertices (or nodes) represent aminoacids, and an edge (or link) is present whenever these two aminoacids are in contact. A network such as this, as we will see throughout the chapter, can give us many hints about the system it is representing. To start with, the network in figure $1 \mathrm{C}$ tells us that this protein is modular. Although we already see that this protein is modular from the examination of its structure, the important point to emphasize is that the network alone mirrors this 

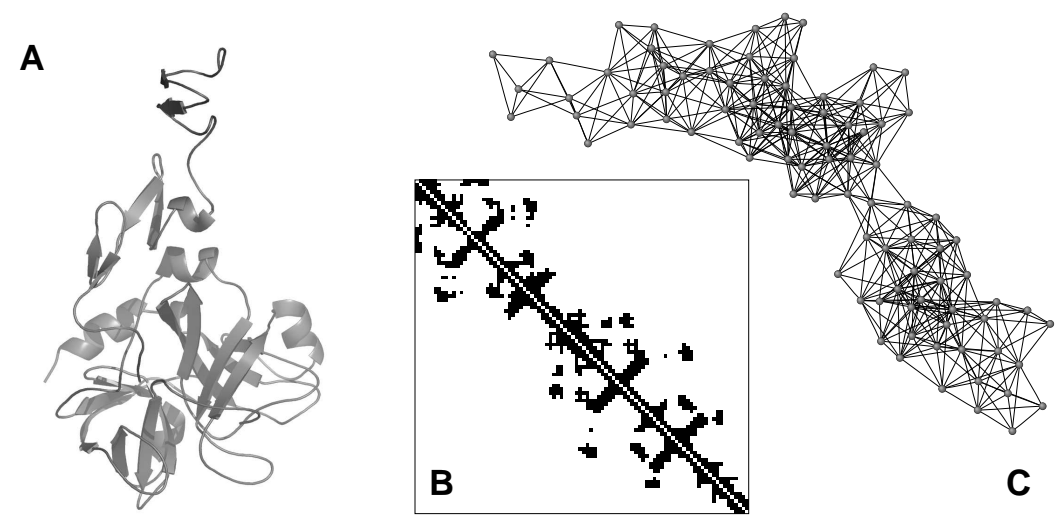

Figure 1: A. A fragment of the structure for the human blood coagulation factor Xa (pdb 1xka). B. The associated contact matrix. Black dots represent above threshold proximity between alpha carbons in the sequence, which are arranged as rows and columns in the order of the backbone $\mathbf{C}$. The associated graph in which nodes represent aminoacids, and links represent contact between them.

modularity, and this is especially relevant whenever we don't have a clear picture of the system for which the network was measured.

Many other properties are in fact measurable just from the networks, which describe other equally interesting properties. Applying various concepts which can be defined on graphs, we can examine the networks of different systems trying to understand their overall features, and we can try to make sense of their connection patterns. In this chapter we will see how some of the properties of complex systems can be approached through the study of the properties of their corresponding networks, the so-called complex networks.

\section{Basic properties of random graphs}

A graph $G$ is defined by a set of $N$ vertices (or nodes) $V=\left\{v_{1}, v_{2}, \ldots, v_{N}\right\}$ and a set of $L$ edges (or links) $E=\left\{e_{1}, e_{2}, \ldots, e_{L}\right\}$, which connect pairs of vertices. Depending on the existence of directionality in the edges, graphs can be either $d i$ rected or undirected. Figure 2 depicts two graphs, one directed and one undirected, using arrows as a representation of directed edges. A certain graph $G$ can, by this simple definition, represent the structure of a given system, in which, nodes correspond to units and edges to interactions. In the following, we will be concerned with graphs in which edges have no weights attached, although many interesting results exist for weighted graphs.

The number of edges $k$ that arrive to vertex $v$ is called degree, and it is divided, in the case of directed graphs into $k_{i}$, the indegree, and $k_{o}$, the outdegree, with $k=k_{i}+k_{o}$, as shown in figure $2 \mathrm{C}$. The average degree is, then, $z=\langle k\rangle=2 L / N$, since every edge is attached to two different vertices. The distance between two 
A

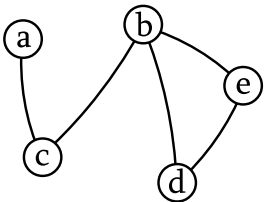

B

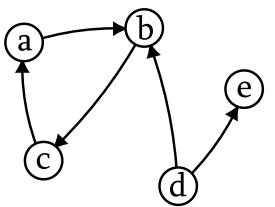

C

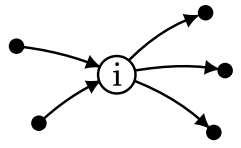

D

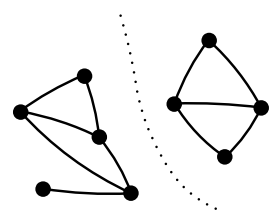

Figure 2: A. An example of undirected graph $G$ with $V=\{a, b, c, d, e\}$ and $E=$ $\{(a, c),(b, c),(b, d),(b, e),(d, e)\}$. B. Example of a directed network with the same $V$ and $E=\{(a, b),(b, c),(c, a),(d, b),(d, e)\} \mathbf{C}$. A node with degree $k=5$ and indegree $k_{i}=2$ and outdegree $k_{o}=3$. D. An undirected graph with two components.

vertices in the graph $d\left(v_{i}, v_{j}\right)$ is defined as the shortest number of links that have to be crossed to reach $v_{j}$ from $v_{i}$, and since we are not dealing with weighted edges, it is an integer number. As an example, in the graph of figure $2 \mathrm{~A}, d(a, e)=3$ and $d(c, d)=2$.

A random graph is a graph in which the pairs of connected vertices have been selected uniformly at random between all the possible pairs of vertices. Basically, this is equivalent to consider that every edge in the graph is present with an independent probability $p$, and absent with probability $1-p$. In fact, there is a whole ensemble of graphs for a single value of $p$, and random graph theory deals with the average properties of these ensembles. The average number of edges in these graphs is thus

$$
\langle L\rangle=\frac{N(N-1)}{2} p,
$$

and accordingly, the average degree is, then $z=\frac{2\langle L\rangle}{N} \approx N p$, the last equality being valid only for large $N$. This kind of random graphs were first studied by Erdôs and Rényi, and many of their average properties are have been solved analytically in the limit for large $N$ [Bollobás, 2001]. Random graphs are important because they represent the null hypothesis about systems that can be modelled as graphs. The study of their properties is thus crucial to understand real graphs, since the comparison between random graphs and real ones tells us what is relevant about the latter.

One of the first interesting aggregate measures to consider is the degree distribution, $P(k)$ or $p_{k}$. This function describes the graph by giving the fraction of nodes that have a certain degree $k$. As an example, we can calculate $p_{k}$ for a random 


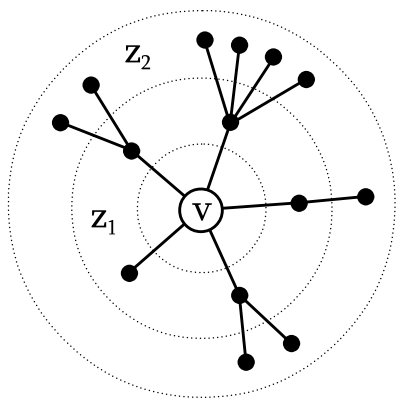

Figure 3: A vertex $v$ surrounded by its first neighbours, on average $z_{1}$, and its second neighbours, on average $z_{2}$.

graph, given the probability of linking two of its vertices, $p$, which is

$$
p_{k}=\left(\begin{array}{c}
N-1 \\
k
\end{array}\right) p^{k}(1-p)^{N-1-k} \approx \frac{z^{k} e^{-z}}{k !} .
$$

The second equality becomes exact in the limit of large $N$, when a finite average degree $z=p / N$ makes $p$ necessarily very small. This is, in fact, the Poisson distribution, an important fact to remember, as we will see.

Depending on the exact organization of the $L$ edges, a certain graph can have different components. Components are defined as the subsets of $V$ in which all vertices of the subset can be reached from other members of the subset, but not from any other vertices. Figure 2D depicts a graph with two components. In random graphs, components have an important role. Basically, as $z$ grows, a random graph shows a phase transition at which the so-called giant component forms.

For small values of $z$, the few edges present in the graph are unable to connect the vertices, and the result is a graph with many small components, having an average size that remains constant with larger $N$. However, for a critical value of $z$, a given fraction $\alpha$ of vertices form a single component, therefore having a size $\alpha N$ which scales linearly as $N$ grows. This is the giant component, and it goes with all the rest of smaller components, which still remain constant in size the graph grows. The appearance of the giant component occurs, in fact, at $z=1$.

\section{Average path length}

Another important average quantity in graphs is the average path length,

$$
\ell=\frac{1}{N(N-1)} \sum_{\forall i, j} d\left(v_{i}, v_{j}\right),
$$

which actually represents the average distance between any pair of vertices. For graphs with given degree distribution, we can calculate $\ell$ in the following way [Newman, 2002a]. Let's consider a certain vertex $v$, such as the one shown in 
figure 3. First, we can calculate the average number of first neighbours, $z_{1}$. This is, in fact, the same as $z$, since the degree measures the number of neighbours of a vertex. Now we can calculate the number of second neighbours, $z_{2}$. To do this, we first need the distribution $p_{k}^{\prime}$ of number of remaining edges of a certain vertex, given that we arrive from one of them, chosen at random.

First, we must take into account the fact that a vertex with more degree has a greater probability of being accessed, i.e., proportional to its degree. Second, we also have to take into account the edge we arrived along, and remove it from the counting. The result is, then,

$$
p_{k-1}^{\prime}=\frac{k p_{k}}{\sum_{i=0}^{N} k p_{i}}
$$

including the proper normalization. On average, then, we will find that following a random edge, the number of remaining edges which we can follow is

$$
\sum_{k=1}^{\infty}(k-1) p_{k-1}^{\prime}=\frac{\sum_{k=0}^{\infty}(k-1) k p_{k}}{\sum_{i=0}^{N} k p_{i}}=\frac{\left\langle k^{2}\right\rangle-\langle k\rangle}{\langle k\rangle} .
$$

That tells us the average of second neighbours found by following one of our first neighbours, but if we follow an average of $k_{1}=k$ first neighbours, we have the total number of second neighbours from vertex $v$, that is,

$$
z_{2}=\left\langle k^{2}\right\rangle-\langle k\rangle
$$

For the case of a random graph, with a Poisson distributed $p_{k}$, we have $z_{2}=\left\langle k^{2}\right\rangle$, so the mean number of second neighbours in the special case of a random graph is just the mean number of first neighbours squared, which doesn't hold in general.

We can now extend this calculation to further neighbours. The distribution $p_{k}^{\prime}$ also tells us how many neighbours a second neighbour has, and in fact it is true for neighbours at distance $m$ in general. In brief, the average number of neighbours at distance $m$ of $v$ is

$$
z_{m}=\frac{\left\langle k^{2}\right\rangle-\langle k\rangle}{\langle k\rangle} z_{m-1}=\frac{z_{2}}{z_{1}} z_{m-1}=\left[\frac{z_{2}}{z_{1}}\right]^{m-1} z_{1} .
$$

Depending of the factor $z_{2} / z_{1}$, the number of accessible neighbours can be infinite, something which reminds us about the phase transition mentioned above. The critical point for this transition is then, $z_{2}=z_{1}$, which in the case of random graphs translates to $z=1$, as already mentioned. In general, though, we can derive an expression for the equation $z_{2}-z_{1}=0$ making use of equation 5 , which gives us

$$
\left\langle k^{2}\right\rangle-2\langle k\rangle=\sum_{k=0}^{\infty} k(k-2) p_{k}=0 .
$$

To return to our previous discussion, we can now calculate the average path length $\ell$ making use of equation 6 . Basically, at some $m$ in equation 6 the number of accessible neighbours will be $N$, and that is precisely $\ell$, since it is the average number 
of steps at which an average node reaches the whole graph. Taking logarithms for equation $N=z_{\ell}$ and reorganizing, we arrive at

$$
\ell=\frac{\log \left(N / z_{1}\right)}{\log \left(z_{2} / z_{1}\right)}+1 .
$$

For the special case of Erdős-Rényi random graph, in which $z_{2}=z^{2}$, the expression reduces to $\ell=\log N / \log z$. This is an important result, in fact. It says that, in random graphs, $\ell$ grows rather slowly with the size of the graph, and that a big graph with $N=1000$ and $z=4$ will have $\ell \approx 5$.

Graphs can also display structure at the local scale, and one of the easiest ways to describe it is by measuring of the average clustering coefficient, or $C$. This coefficient is a measure applied to a given vertex $v_{i}$, and it measures how far a vertex is of being part of a clique. A clique is basically a small graph with full connectivity, every vertex is connected to every other. It is clear that if a certain vertex $v_{i}$ is part of a clique, the number of edges between its $k_{i}$ neighbours must be $k_{i}\left(k_{i}-1\right) / 2$. If instead of that we find $E_{i}$ edges, the corresponding fraction is precisely $C_{i}$, the clustering coefficient, that is,

$$
C_{i}=\frac{2 E_{i}}{k_{i}\left(k_{i}-1\right)} .
$$

When $C_{i}$ is average for the whole network, we have

$$
C=\frac{1}{N} \sum_{k=0}^{N} C_{i}=\frac{1}{N} \sum_{k=0}^{N} \frac{2 E_{i}}{k_{i}\left(k_{i}-1\right)} .
$$

The interest in clustering came from social networks, in which networks of acquaintances usually display a high degree of clustering: your friends tend to be friends of each other. This is in contrast to random graphs in which clustering is very small. Actually in random graphs, since each vertex is active with probability $p$, the clustering is always the same, that is $C=p=\langle k\rangle / N$. So clustering is very, very small for large graphs.

\subsection{Small-worlds}

Now that we have seen both the average path length and the property of clustering, we can use them to assess if a certain graph is "small world". The small-world effect was demonstrated in the 1960 s by Stanley Milgram in a famous experiment involving letter passing. He gave some letters to some friends of his, asking them to pass the letters to a friend of theirs that they thought was closest to the recipient in the letter. Contrary to logic, the number of steps, or friendships, required to reach the recipient was rather low, in the order of 6 or 7 . But how could this happen when social networks have a very high degree of clustering? Although the average path length for a random graph goes as $\log (N) / \log (z)$, in a very clustered network the average path length must be larger perforce.

For instance, let's consider the network in figure 4A. It's a highly clustered network. In particular, the clustering coefficient $C$ of this network is precisely 

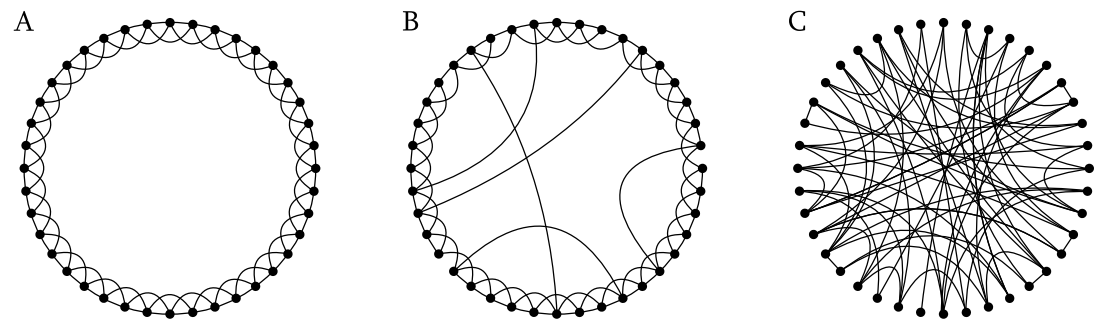

Figure 4: A. An example of a very clustered network with the topology of a one dimensional space. To reach any vertex from any other, many links have to be crossed since all connections are local. B. The same network as in A with some edges rewired at random. C. A completely random network.

$1 / 2$ since all nodes are equivalent and the 4 neighbours of each node have 3 of the 6 possible connections between them. Having 40 nodes, the clustering of an equivalent random network would be $4 / 40=0.1$ so this network has 5 times more clustering. We could think of this network as a crude model of a social network.

But an important ingredient is missing. Although in a social network many of one's friends are friends themselves, there is a very small fraction of "longdistance" friends, removed from the local community of acquaintances, that represent something similar to the "random" links of an Erdôs-Rényi graph. As we will see, this small number of edges alone provide enough long-range jumps to make the average path length fall rapidly, and at the same time they don't affect the clustering very much, the so-called small-world effect.

The small-world effect was studied in [Watts and Strogatz, 1998] using a model which considers precisely the network of figure 4A. Starting with it, a rewiring process is defined which takes each edge with probability $p$ and rewires it to a random vertex. In this way, we have a graph which depends on $p$. If we plot the normalized clustering $C(p) / C(0)$, and at the same time the normalized average path length $L(p) / L(0)$ the result is what is shown in figure 5. As it is apparent, there is a broad region of $p$ values (very small values, noting that the 5 axis is logarithmic) in which clustering is still high whereas the path length is already low. The explanation for this is found in the very small fraction of connections that connect remote parts of the system reducing the average path length to be comparable to that of a random graph.

\section{Protein structure and contact graphs}

At the smaller scale, we can consider proteins as a key example of a complex system composed by many parts in interaction (the protein residues). As is well known from biochemistry and molecular biology, proteins play a key role in cell function and they actually are responsible for many different features of cell behavior, from shape to communication [Albert et al., 2002]. Here structure and function are intimately linked. From the linear chain of residues coded at the genome sequence 


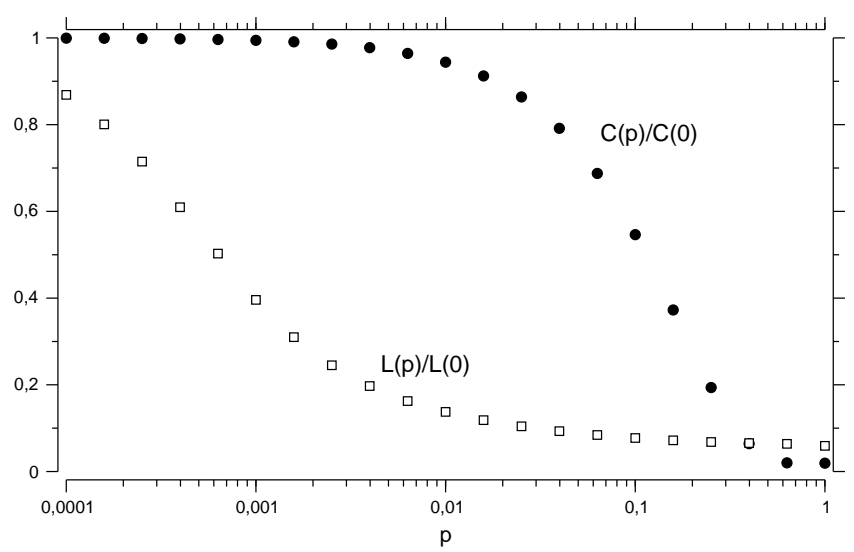

Figure 5: The small-world effect quantitatively. The graph shows the normalized clustering coefficient and the normalized average path length as a function of the probability of rewiring $p$. Note that the $p$ axis has a logarithmic scale. For a very small value of $p$, around 0.01 , the average path length has dropped substantially whereas the clustering coefficient is still almost untouched. The joint appearance of these two properties describe the small-world effect. The graphs used had $N=$ 1000 and $\langle k\rangle=10$, replicating the results in [Watts and Strogatz, 1998].

level, the chain acquires a functional shape by folding in three dimensions towards the so called native state. The final shape defines a higher-order structure in relation with the linear sequence and thus involves further, long-range interactions among residues.

Since the contact graph of aminoacid interactions defines a complex network, we might first ask what type of overall pattern is found here. Folding is known to be highly optimized in relation with what would be expected from a polymer exhibiting random interactions among residues [REF GENERAL??]. Instead, protein architecture seems to display a number of features resulting from a selection process [Gutin et al., 1995]. Using the previous tools of graph analysis, we can first explore the question of the presence of small world behavior in the protein contact graph.

As shown in figure 3, the statistical pattern of organization of protein contact graphs reveals a well-defined small world topology. Clustering is typically much higher than expected from random wiring (for which we should observe $C \sim N^{-1}$ ) and the average path length scales with the logarithm of system's size $N$. It is interesting to see that we actually are playing with a scenario of graph rewiring not so far from the Watts-Strogatz model. Staring with a linear chain of residues connected to only two nearest neighbors, protein folding involves the creation of a number of shortcuts which we can easily identify to those key residues responsible for the folding.

Beyond these global features, which indicate that some quantitative traits can be properly identified, other relevant properties can be measured, perhaps much 

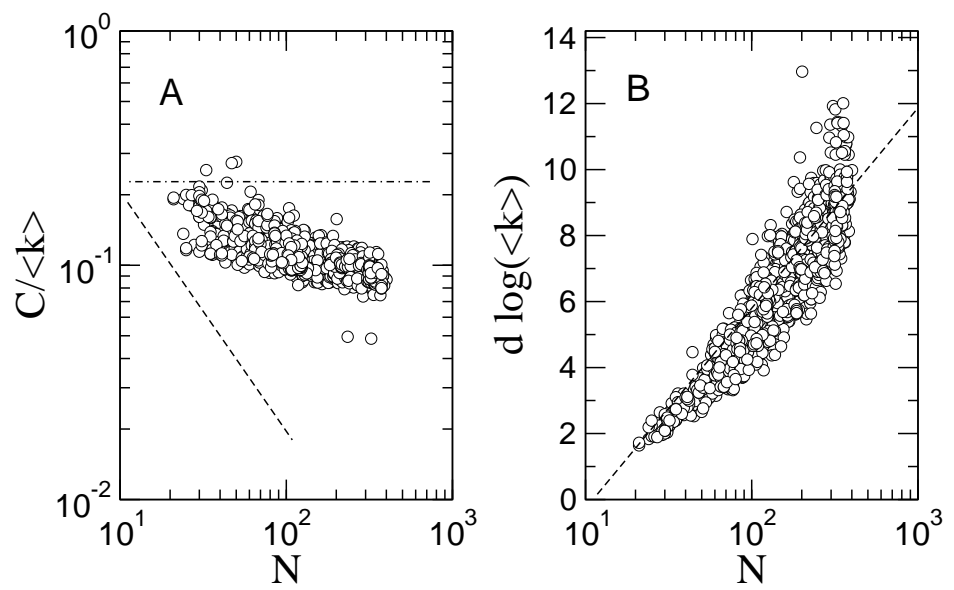

Figure 6: Small world structure of protein folding maps. Here the two key global measures are shown for a large number of proteins from the *** database (REF). In (a) the clustering coefficient is shown (properly normalized with the average degree). The predicted scaling relation from a random graph (i. e. $C \sim N^{-1}$ ) is indicated as a dashed line. In (b) the average path length is plotted against size. The prediction from a small world architecture indicate that $l \sim \log N / \log z$. This is indicated as a dashed line, which is closely followed by the observed data set.

closer to the functional characterization of protein graphs and other complex networks. Modular patterns can be observed by looking at how subsets of a given graph are connected among them. In its simplest terms, modules can be seen as groups of units which are more connected between them than with other parts of the graph. Modules have been found in biological systems at multiple levels, from RNA structures [Ancel and Fontana, 2000] to the cerebral cortex (see [Solé et al., 2002a]). The widespread character of modular organization has been always associated to functionality, compartmentalization and evolution. The evolutionary conservation of modules is well known from different examples, particularly in in early development [von Dassow et al., 2000, von Dassow and Munro, 1999, Solé et al., 2000]. The argument is that the special features of some of these modules are tightly linked to their robustness under different sources of noise. As such, they would be the units of selection.

The modular character of biological networks is assumed to be a consequence of both their robustness and evolvability [Wagner, 1996, Wagner and Altenberg, 1996]. In a different context, it has been suggested that modularity might arise from the intrinsic structure of the non-metric mapping between genotype and phenotype, at least in molecular networks [Stadler et al., 2001]. Although functionality must pervade the selection of some modular structures, we will see below that proto-modules might actually result as emergent patterns without any predefined functional meaning. 

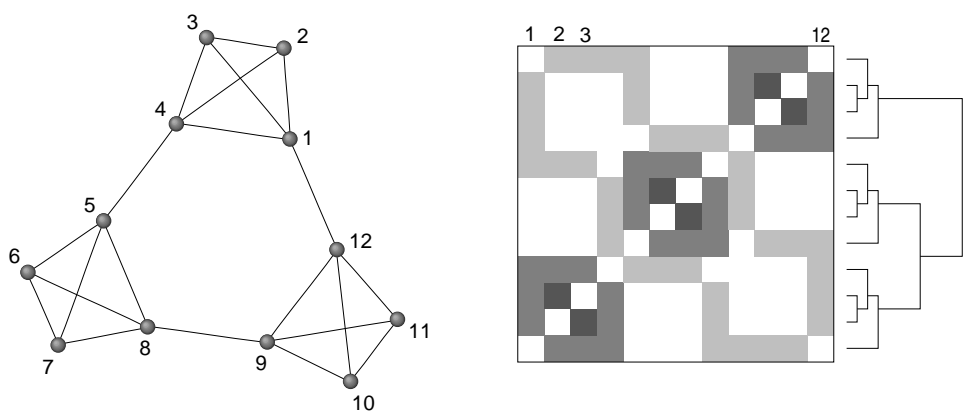

Figure 7: Left. A simple graph with 3 modules Right. The modular structure as viewed using hierarchical clustering on the topological overlap matrix.

\section{Hierarchical clustering in contact maps}

Although there is no agreed measure of overall modularity for graphs, the intuitive notion that a module is a group of nodes that have higher connectivity to the inside than to the outside seems sensible, and some simple heuristic algorithms can reveal this straightforwardly [Ravasz et al., 2002]. To do so, the topological overlap (TO) of two nodes has to be defined. The degree of TO of two vertices tries to give a value in the range $(0,1)$ for the fact that two vertices belong to the same "module". If they do, they will probably have many neighbours in common, and that is precisely what the TO value measures. Its definition is

$$
O\left(v_{i}, v_{j}\right)=O\left(v_{j}, v_{i}\right)=\frac{J\left(v_{i}, v_{j}\right)}{\min \left(k_{i}, k_{j}\right)}
$$

where $J\left(v_{i}, v_{j}\right)$ denotes the number of nodes to which both $v_{i}$ and $v_{j}$ are linked, plus one if there is a direct link between them. The OT value is commutative, and can be seen as a measure of the proximity in terms of modules of two vertices. Given the matrix of OT values, we can apply the general algorithm for hierarchical clustering, which tries to group together those vertices in the system that have a high proximity, that is, high TO. Basically, this algorithm starts with a matrix with the original values, and repeatedly finds the highest value, grouping together the corresponding row and column (the two vertices) into one new aggregated vertex, and computes the new values for the grouped vertices, so as to be able to apply the same procedure to the resulting matrix until it collapses into a single value.

The result of this process is a tree, and also an ordering of the vertices of the graph, which enables us to display the topological overlap in a suitable way, as figure 7 shows. On the left there is a graph with evident modular structure, at the right, the corresponding output of the hierarchical clustering algorithm. Values between 0 and 1 have beep mapped to levels of gray. As shown, the indices in the graph correspond to the indices in the matrix, so for example, vertices 2 and 3 have a higher TO than others, and hence are in the central part of the modules, with a darker value. Modules in this diagram are organized along the darker regions of the 

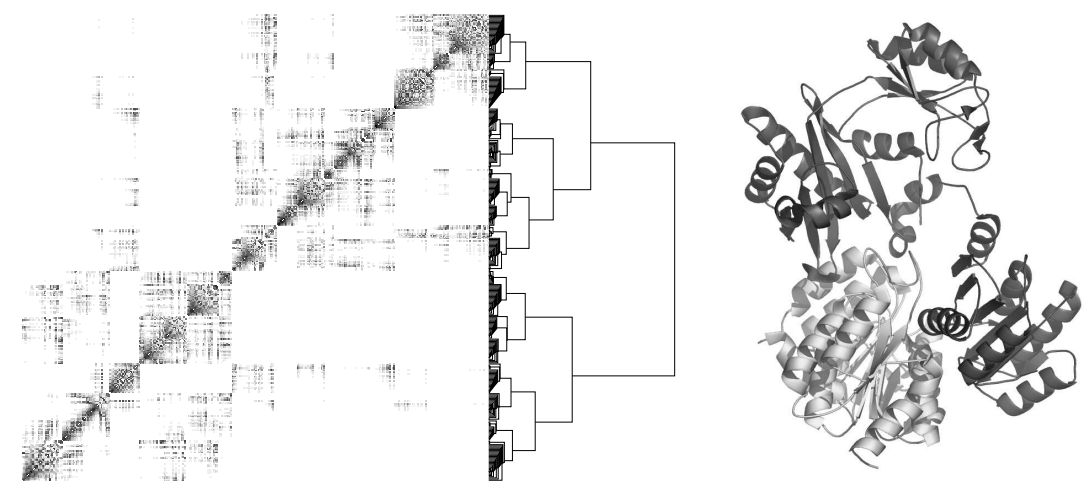

Figure 8: Left. Hierarchical organization of the Succinyl-CoA synthetase from the pig (Sus scrofa), as uncovered by the hierarchical clustering algorithm (see text). This method reveals two basic modules (which correspond to the two chains A and B) which also include a lot of internal substructure, showing a nested hierarchy of folding structures. Right. The corresponding protein structure (PDB code is 1euc).

diagonal, because mainly the algorithm has separated them in the linear ordering of the matrix. That is apparent in the tree that appears on the right.

What is the pattern displayed by protein contact maps in terms of hierarchical clustering? An example is shown in figure 8 . We can clearly appreciate the presence of two well-defined modules, which are actually mapped into the two chains playing a functional role in this particular protein. An interesting feature that becomes obvious from the previous plot is that we actually have a rather complex, nested pattern of modularity: groups of proteins appear more connected at different scales and belong to larger structures. Modularity is thus associated with hierarchical organization. This might actually be related with the fact that the process of folding is hierarchical. The nested structure of the overlap map would be a fingerprint of the hierarchies involved in the folding process. This method is able to identify the presence of well-defined domains in terms of topological arrangements, but can be used in the analysis of any other network structure. As we will see, modularity is actually a preeminent feature of the organization of complex networks.

\section{Protein interaction networks}

It is often said that the actions and properties of each cell are basically determined by the proteins it contains, which implement, like complex nanomachines, the tasks needed by the cell. In this constantly changing chemical world, proteins seldomly work alone. By and large, almost all proteins are part of protein complexes, or at least engage in some form of interaction with other proteins. By means of physical contacts, proteins enable the cell to actively build structures, process signals from 

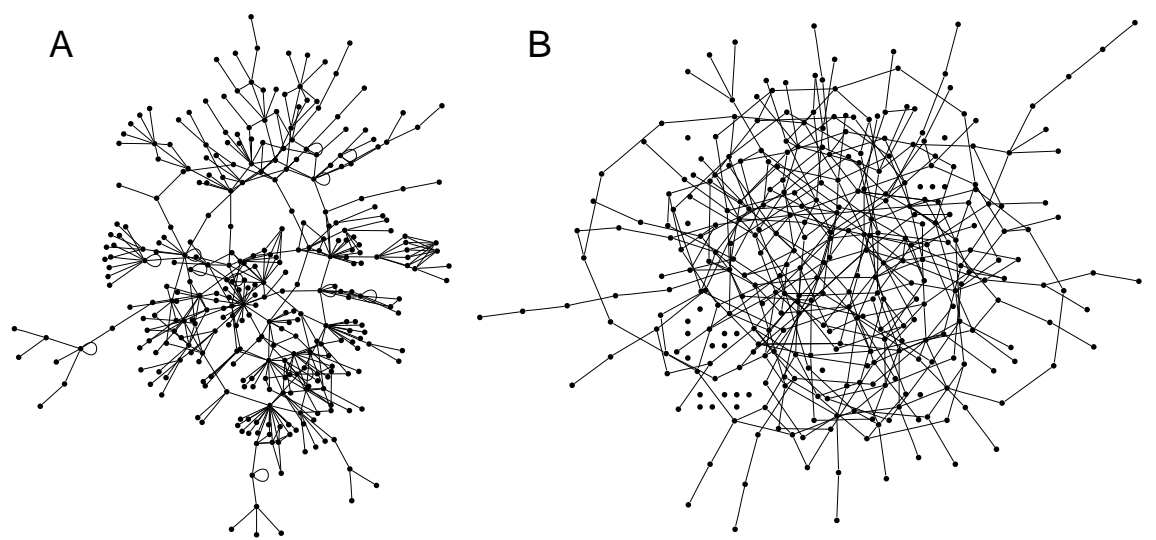

Figure 9: A. The largest component of the known portion of the protein-protein interaction network of Homo sapiens. It is easy to see that this network is far from random. The majority of proteins interact just with a very limited number of other, such as 1 or 2, whereas a few proteins interact with tens of others. B. A random graph with the same number of nodes and links that the network A, for comparison.

the environment, redirect chemicals to different metabolic pathways, or form the basis of gene regulation. A very useful picture of the organization of the cell can be obtained, therefore, from the information of which pairs of proteins interact with each other. By means of this information, a graph can be constructed, which describes the innerworkings of the whole cell. However, the simple examination of the networks is not very useful, given their size. It is when we investigate this graph with the tools of graph theory that some interesting properties become clearer.

In figure 9A a part of the proteome network of Homo sapiens is shown. This network is one of the smallest in the DIP database. Although not especially useful as a detailed map, the network displays, nevertheless, many interesting properties. In comparison with 9B, which is a random network with the same number of vertices and edges, the most important feature can readily be observed: the heterogeneity in the degree of vertices. Whereas the random network has a more or less homogeneous distribution, the proteome network shows a huge number of proteins with a few connections, and at the same time, certain proteins have a very big number of connections, the so-called hubs. Other features can be distinguished right away, such as for example, the existence of groups of nodes that connect to two hubs at once, forming a kind of [abanico].

To quantify the mentioned heterogeneity, the distribution of degree is shown, for the bigger proteome network of Saccharomyces cerevisiae, in figure 10. Although many functions would qualify as degree distributions with a big number of low degree nodes and a small number of very high degree ones, the precise functional form of the distribution is a strict power-law of the form $P(k) \sim k^{-\gamma}$ with exponent between 2.1 and 2.5 (for different measuring methods and organisms, as shown in figure 10). The networks in this case are much bigger, and hence, they 

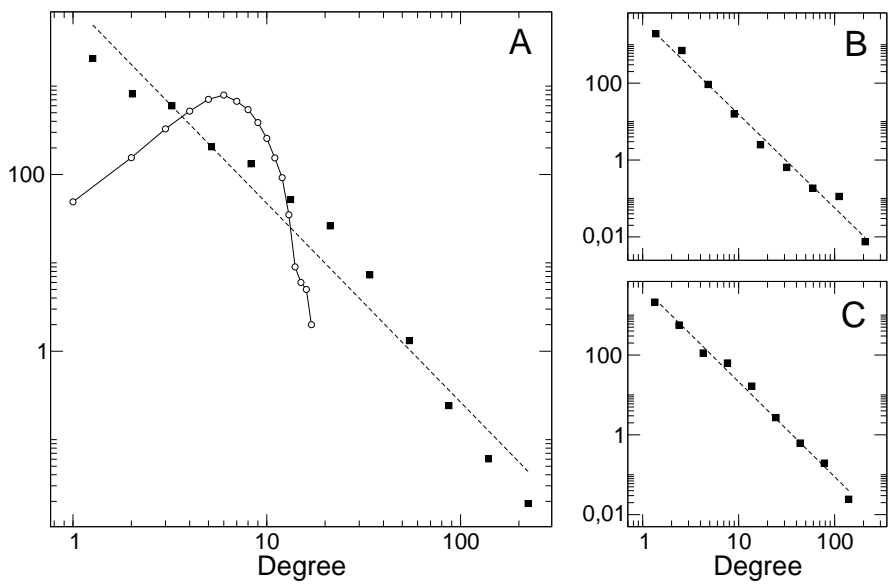

Figure 10: A. In black squares, degree distribution of the proteome graph of Saccharomyces cerevisiae taken from the DIP database and in white circles a random graph with the same number of vertices and edges. Note that axes are logarithmic, revealing that the proteome graph has a power-law degree distribution, that is $P(k) \sim k^{-\gamma}$. B $S$. cerevisiae from [Ito et al., 2001] $(N=3280, L=4549$ and $\gamma=2.43 \pm 0.10)$. C C. elegans taken from [Li, S. et al., 2004] $(N=3228$, $L=5625$ and $\gamma=2.37 \pm 0.08$ ). To propery average the power laws, the bins were taken logarithmically distributed.

have a broader distribution. The message is, then, clear enough: since the two distributions are hardly comparable, random networks seem to be poor models of real protein interaction networks. This is actually a more general result, which has been discovered in networks of very diverse fields [Albert and Barabási, 2002, Dorogovtsev and Mendes, 2003].

To support the deviation from a purely random graph there is the fact that the proteome network is a small-world, since the comparison of its properties with those of a random graph of its size, that is,

$$
\begin{array}{crl}
C_{\text {prot }}=0.143 & L_{\text {prot }} & =4.218 \\
C_{\text {rand }}=0.00139 & L_{\text {rand }} & =4.515,
\end{array}
$$

in fact satisfy $C_{\text {prot }} \gg C_{\text {random }}$ and $L_{\text {prot }} \approx L_{\text {random }}$ (for this calculations the DIP dataset was used).

The other mentioned feature seen in the graph of figure 9A concerns de correlation between the degree of vertices, which seems to account for the [abanicos] in the graph. Given two connected vertices in a network, we can simply ask what is the correlation between their degrees. In other words, assuming a certain degree distribution $p_{k}$ to dictate the degrees of the vertices in an otherwise random network, we would expect the joint probability distribution of the remaining degrees of vertices $e_{j k}$ to be $q_{j} q_{k}$, the simple product of remaining degrees. If that is not the value found, we can measure the difference by averaging through all the edges 
in the graph, that is, $\langle j k\rangle-\langle j\rangle\langle k\rangle=\sum_{j k} j k\left(e_{j k}-q_{j} q_{k}\right)$. This sum measures the assortativeness of a network [Newman, 2002b]. If it is positive, high degree nodes tend to connect to other high degree nodes, whereas a negative value describes the opposite, high degree nodes will preferentially be neighbours of low degree ones.

To properly compare different networks, it is convenient to normalize the sum by the maximum value attainable, so that the result is in the range $(-1,1)$. The maximum value, in fact, is present when the joint distribution $e_{j k}$ is simply $q_{k} \delta_{j k}$, that is, when nodes of a given degree just connect to nodes of the same degree. It is easy to see that the result is $\sum_{k} k^{2} q_{k}-\left[\sum_{k} k q_{k}\right]^{2}$ which is the variance of the remaining degree distribution, $\sigma_{q}^{2}$. Hence, the normalized correlation function is

$$
r=\frac{1}{\sigma_{q}^{2}} \sum_{j k} j k\left(e_{j k}-q_{j} q_{k}\right) .
$$

When calculating this value for an actual network with known values, we can use the following formula [Newman, 2002b],

$$
r=\frac{L^{-1} \sum_{i} j_{i} k_{i}-\left[L^{-1} \sum_{i} \frac{1}{2}\left(j_{i}+k_{i}\right)\right]^{2}}{L^{-1} \sum_{i} \frac{1}{2}\left(j_{i}^{2}+k_{i}^{2}\right)-\left[L^{-1} \sum_{i} \frac{1}{2}\left(j_{i}+k_{i}\right)\right]^{2}}
$$

where $j_{i}, k_{i}$ are the degrees of the vertices at the ends of the $i$ th edge, with $i=$ $1 \ldots L$. When we apply this measure to protein interaction networks, the results are clear, protein networks are disassortative, that is, hubs connect with high correlation to low degree nodes. The results are shown in table XXXXXX.

However, the assortativeness of a network gives just a global description of the correlations in connectivity. If we want more detail in this type of analysis, we have to turn to other techniques. One more time, measuraments will involve the comparison of the correlations of a given graph with its randomized counterpart [Maslov and Sneppen, 2002, Maslov et al., 2002]. Since we are comparing degree correlations, though, it is important in this case to create a random graph that has the same degree distribution.

This is achieved through a rewiring process, in which the degree distribution is preserved. If two edges are chosen at random that do not have vertices in common, the simple exchange of their starting vertices will give a graph with the same degree distribution but otherwise random. Iterating this process as many times as twice the number of edges yields a reasonably randomized graph, with preserved degree distribution. If we call $P\left(k_{0}, k_{1}\right)$ the probability of finding an edge connecting two nodes with degree $k_{0}$ and $k_{1}$, and $P_{r}\left(k_{0}, k_{1}\right)$ the random equivalent, we can measure two things, that is,

$$
R\left(k_{0}, k_{1}\right)=\frac{P\left(k_{0}, k_{1}\right)}{P_{r}\left(k_{0}, k_{1}\right)},
$$

whose deviation manifest the correlations, and

$$
Z\left(k_{0}, k_{1}\right)=\frac{P\left(k_{0}, k_{1}\right)-P_{r}\left(k_{0}, k_{1}\right)}{\sigma_{r}\left(k_{0}, k_{1}\right)},
$$


A

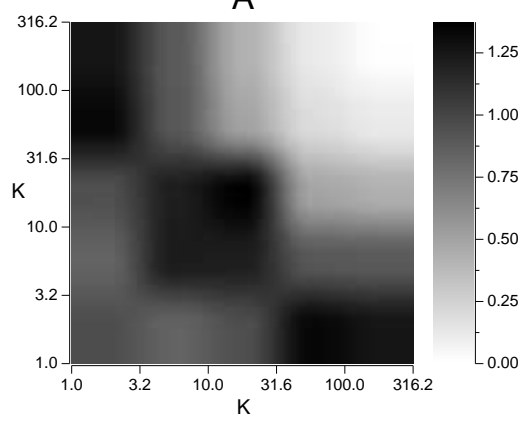

B

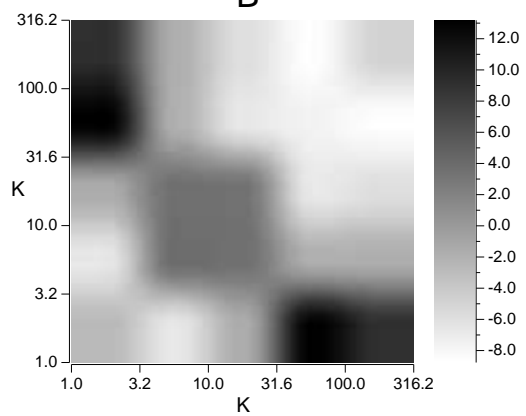

Figure 11: A. Correlation profile of the protein interaction network, with the dataset taken from [Ito et al., 2001]. The value $R\left(k_{0}, k_{1}\right)$ is shown. B. Same as A, but showing the Z-score, $\mathrm{Z}\left(k_{0}, k_{1}\right)$.

quantifying the statistical significance of $R\left(k_{0}, k_{1}\right)$, or Z-score. The value $\sigma_{r}\left(k_{0}, k_{1}\right)$ is the standard deviation of $P_{r}\left(k_{0}, k_{1}\right)$ in an ensemble of randomized networks.

The results of this process are shown in figure 11. The image represents a log-binned matrix in which black indicates a higher value, as the side bar shows. This profile reveals, with some more precision, the disassortative nature of protein interaction networks.

To finish our analysis of the proteome network, we can see its modularity, which is something not apparent in the graph of figure 9A, as we mentioned in the introduction. In the last section we saw that hierarchical clustering can give a clear picture of modularity lacking any good measure of it. A network such as the one shown in 9A has a modularity that is revealed by the hierarchical clustering shown in 12 (bottom). The random network of 9B is also displayed for comparison (top).

\subsection{Proteome model}

Since we now know the structure of the proteome network, we can ask ourselves how did the proteome network emerge. In principle, we can try to find simple mechanisms that we know alter the relationship between proteins in the genome and try to interpret them in terms of the rewiring process that affects the protein interaction network. A simple mechanism does, in fact, exist, and it gives a very plausible answer to the question of the origin of the proteome network.

The genome grows mainly due to gene duplication. From time to time, a gene is duplicated in the cell replication process that gives rise to a redundant copy of a gene. After a while, mutations accumulate that make the two copies diverge pushing their sequences apart, and as well the functionality of the proteins they code for. The result is that most genes currently present in the genome can be traced back to ancient duplications of other genes. Since proteins are the product of genes, we can model gene duplication in the way figure 13 shows, which were used in the model analyzed in [Solé et al., 2002b]. 

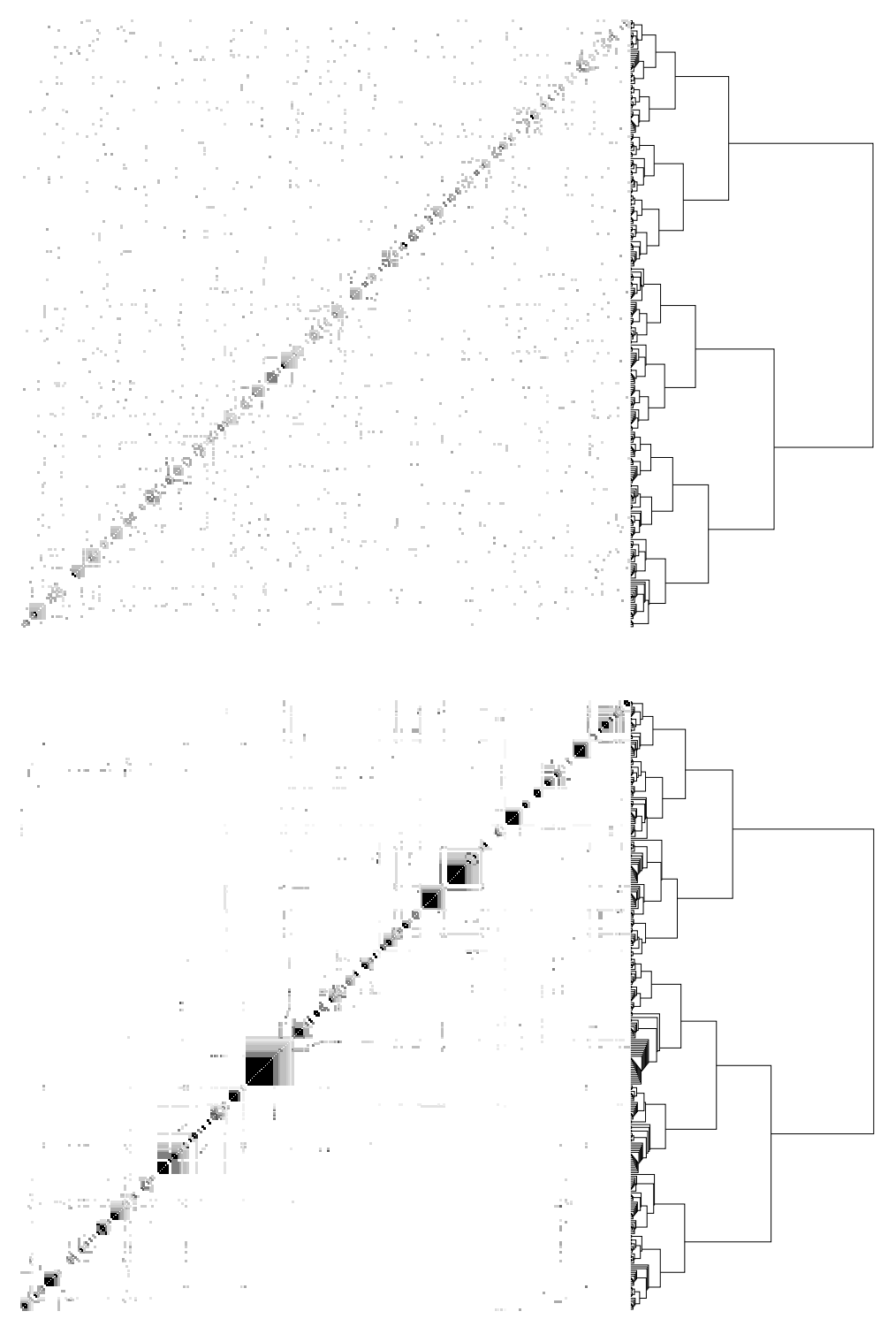

Figure 12: Bottom. Modular appearance of the network of figure 9A. Top. The result of applying the same algorithm to a random network of about the same size. 
We start form a set $m_{0}$ of connected nodes, and at each time step we perform the following operations

(i) One node of the graph is selected at random and duplicated

(ii) The links emanating from the newly generated node are removed with probability $\delta$.

(iii) New links (not previously present after the duplication step) are created between the new node and all any other node with probability $\alpha$. Although available data indicate that new interactions are likely to be formed preferentially towards proteins with high degree here we do not consider this constraint.

Step (i) implements gene duplication, in which both the original and the replicated proteins retain the same structural properties and, consequently, the same set of interactions. The rewiring steps (ii) and (iii) implement the possible mutations of the replicated gene, which translate into the deletion and addition of interactions with different proteins, respectively. The process is repeated until $N$ proteins have been obtained.

Another model uses very similar rules [Vázquez et al., 2003], but introduces some relevant differences. Duplication is also followed by two probabilistic rules which operate independently. The first (ii) is link deletion. For each of the nodes $p_{j}$ linked to the two $p_{i}$ and its duplicate $p_{i}^{\prime}$, we choose randomly one of the two links $\xi_{j i}, \xi_{j i}$ and remove it with probability $\delta$. Additionally, a new interaction connecting the two proteins (the parent and the duplicated) is introduced with probability $\rho$. The last rule will naturally increase the number of triangles in the system and thus provide a source of high clustering.

The rewiring process seems to be more appropriately defined, since the removal of one of the alternative links allows to "conserve" the function that was somehow present before the duplication event. In Solé's model, the whole set of links of the duplicated gene are preserved and loss of connections affects only the new copy. By using Vázquez's approach, more flexibility is allowed and the interaction map is more likely to remain connected. As defined, it is important to note that duplicates will diverge only to some extent: if a duplication occurs in a gene with degree $k_{i}$, only $\delta k_{i}$ will be removed on average.

The two models collapse into a single mean field description where the average connectivity follows the dynamics

$$
\frac{d K_{n}}{d n}=\frac{1}{n}\left(K_{n}+\phi_{\alpha}\left(n, K_{n}\right)-2 \delta K_{n}\right)
$$

where $\phi=2 \alpha\left(n-K_{n}\right)$ in Solé's model and $\phi=2 \alpha\left(n-K_{n}\right)=\rho$ in Vázquez's model. Actually, in a previous paper [Solé et al., 2002b] we showed that in order to have convergence in the system towards a scale-free stationary distribution we need a very small rate of link addition (which is consistent with observations). If we assume that $\alpha \sim O(1 / n)$ then a single link is added on average each step and 


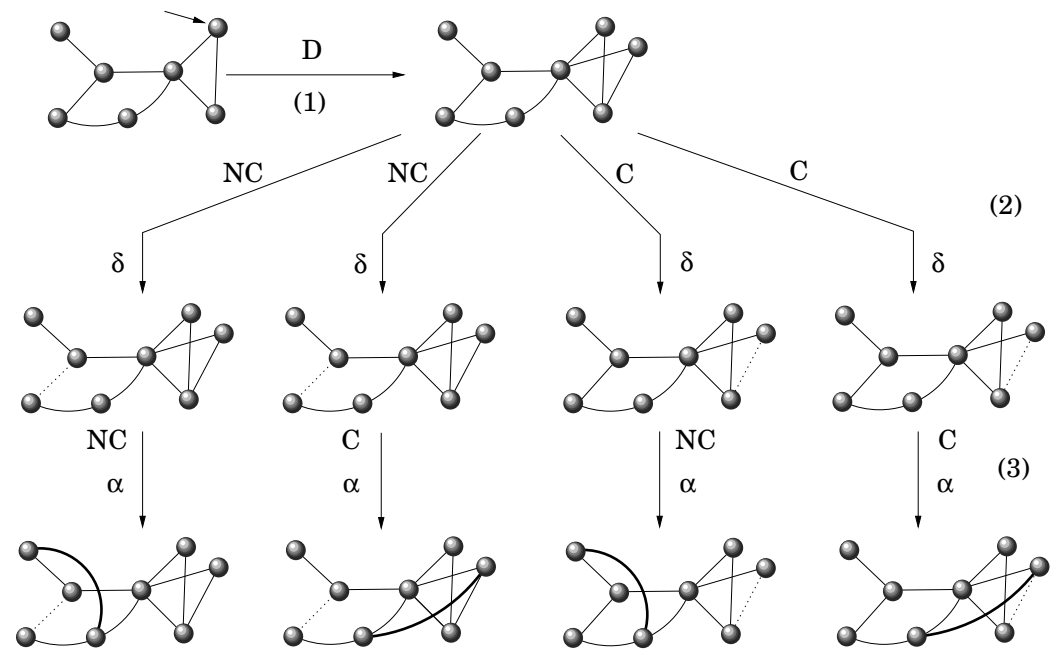

Figure 13: Rules of proteome growth in the four possible scenarios. First, (1) duplication occurs after randomly selecting a node (small arrow). Then (2) deletion of connections occurs with probability $\delta$. This event can be correlated (C) when the deleted links are connected to the newly generated node or uncorrelated (NC), when all links are considered for deletion. Finally (3) new connections are generated with probability $\alpha$, again in a correlated or uncorrelated way. The time scales at which different events occur are known to be very different: duplication takes place at a much slower rate, whereas rewiring is much faster. Additionally, the specific rates at which each event occur might involve preferential attachment to proteins of higher connectivities. All these variants can be included. 
thus the two models are identical in the low-addition limit. Specifically, if the graph is sparse, we have $\alpha\left(n-K_{n}\right) \approx \rho$, which results in a dynamical equation

$$
\frac{d K_{n}}{d n}+\frac{2 \delta-1}{n} K_{n}=\frac{2 \rho}{n}
$$

which has an associated general solution

$$
K_{n}=e^{-\eta(n)}\left(2 \rho \int \frac{e^{\eta(n)}}{n} d n+C\right)
$$

where $\eta(n)=\int(2 \delta-1) d n / n=(2 \delta-1) \ln n$.

This gives

$$
K_{n}=\frac{2 \rho}{2 \delta-1}+\left(K_{0}-\frac{2 \rho}{2 \delta-1}\right) n^{-(2 \delta-1)}
$$

if $\delta>\delta_{c}=1 / 2$, the previous system converges to a graph with a finite average degree

$$
K_{\infty}=\lim _{n \rightarrow \infty} K_{n}=\frac{2 \rho}{2 \delta-1} .
$$

Otherwise, the average connectivity will be $K_{\infty} \rightarrow \infty$. The critical removal rate $\delta_{c}=1 / 2$ thus defines a phase transition separating a phase with a highly-connected system $\left(\delta<\delta_{\mathcal{C}}=1 / 2\right)$ from a sparse phase $\left(\delta>\delta_{\mathcal{C}}\right)$ where a finite number of links will be observed. At this phase, the network becomes fragmented into many components. It is interesting to note that, under the present conditions, the long-term behavior of the average connectivity does not depend on the rate of link addition. What is really important is that the rate of link addition and link removal are similar, so that $\langle k\rangle$ can reach a stationary value. Moreover, it can be shown that although no explicit preferential attachment is included here, the multiplicative nature of the process (in which proteins having more links are more likely to have them copied) actually leads to an effective preferential attachment [Vázquez, 2003].

We can test this prediction by studying the behavior of the model under different rates of link deletion. In order to measure the impact of this rate on network's architecture, we use two different, but closely related measures: (1) the normalized largest component size $S$ and (2) the average, normalized component size $\langle s\rangle$. If $\mathcal{C}(\Omega)=\left\{\Omega_{1}, \Omega_{2}, \ldots, \Omega_{c}\right\}$ is the set of connected components (subgraphs) of the proteome map, so that

$$
\Omega=\bigcup_{i=1}^{c} \Omega_{i}
$$

and $n_{i}=\left|\Omega_{i}\right|$ indicates their size (with $\sum_{i} n_{i}=N$ ), we define

$$
\begin{aligned}
S & =\frac{1}{N} \max \left\{n_{i}\right\} \\
\langle s\rangle & =\frac{1}{N}\left(\frac{1}{c} \sum_{i=1}^{c} n_{i}\right)
\end{aligned}
$$



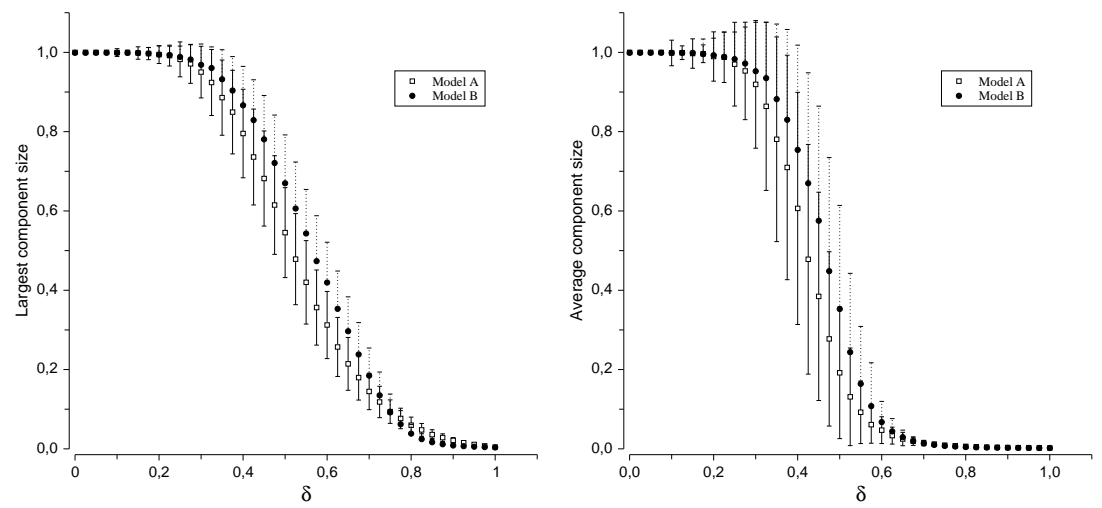

Figure 14: Phase transition in the genome growth models. Here $N=10^{3}$ and averages have been performed over $R=10^{3}$ replicas. Here the size of the largest component and the average size are shown against the rate of link removal $\delta$. The predicted phase transition occurs at $\delta_{c} \approx 0.5$. Due to the finite (small) size of our networks, the transition appears to be less sharp than expected.

In figure 14 we display the two measures against $\delta$ for a $N=10^{3}$ protein network. Close to $\delta_{c}$ we can appreciate a clear change. The two phases are clearly identified, with the connected one showing $S \approx 1,\langle s\rangle \approx 1$ and the fragmented phase showing $S \approx 1 / N,\langle s\rangle \approx 1 / N$. In figure 14 (left) we can see that $S$ decreases slowly close to $\delta_{c}$, where only about half of the nodes remain connected within the largest component. The sharpness of the transition becomes much more obvious in 14 (right). Here we clearly appreciate the impact of rewiring on network's structure, indicating that a large fraction of the overall network structure is formed by small, isolated components. In figure 15 we can see some examples of the graphs generated (largest components) obtained at different rates of deletion.

\section{Gene networks}

The proteome network of last section demonstrates the importance of models that try to capture essential ingredients in network evolution. We have seen the networks implicit in protein and RNA structure, the networks that these molecules form when interacting within the cell as numerous complexes, but we are still lacking one important ingredient, and that is function. Although illuminating, previous analyses dealed only with topological properties, which can describe cells only partially. If we really want to understand cellular functioning, we must turn towards its function, and in particular, to regulatory networks.

Since long ago it is known that genes interact with one another. This interaction is due to the fact that some proteins (transcription factors) can bind to DNA and alter transcription of other proteins, modulating their concentrations. This regulation allows us to model a genome as a network: each gene is mapped to a node, 


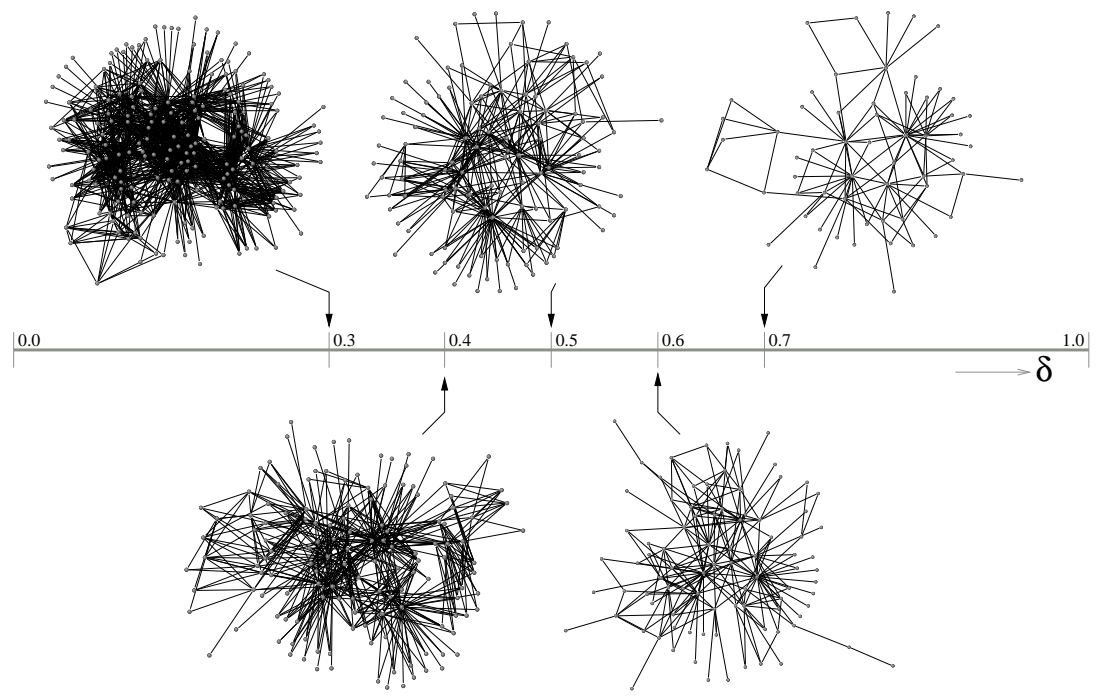

Figure 15: The architecture of the proteome map, as generated by the simple model for different values of the deletion rate $\delta$. As predicted by the mathematical model, two well-defined phases are present. For the first, when $\delta<\delta_{c}=0.5$, the protein map is highly connected and most elements have links to others. Conversely, for $\delta>\delta_{c}$ the graph is fragmented into many components and many components have no links or belong to small isolated subnets. Close to the transition domain, we have a sparse graph with the statistical features displayed by the real proteome map. Such graph displays modular organization, in spite of a complete lack of functionality in the definition of the model rules. 
and regulatory interactions are mapped to the edges between them. Since the existence of regulatory interaction from gene $a$ to $b$ doesn't necessarily imply also that $b$ regulates $a$, we are in fact considering a directed network.

To be able to model this kind of networks, we can simplify things by considering a discretized version of a genome: although gene expression is known to be continuous, we can disregard this and consider genes Boolean. This approximation was pioneered by Kauffman more that 30 years ago [Kauffman, 1969]. This type of analysis, although to some purposes too simple, has been successful in models of genetic circuits [Albert and Othmer, 2003, Solé et al., 2003]. In this kind of modeling, a gene is just like a switch, which can be turned on or off, that is, expressed or not expressed. The nodes in the network are then represented by a set of Boolean variables $\left\{\sigma_{1}, \sigma_{2}, \ldots, \sigma_{N}\right\}$, which are, in fact, functions of discrete time. To determine the value of each gene in the next time step, we will use the values of the inputs of this gene, i.e., the values of the genes that regulate it, and to combine the diverse values of the inputs of a gene, a general function $f_{i}$ is assumed to operate. In general, then, the dynamics of gene $\sigma_{i}$ is

$$
\sigma_{i}(t+1)=f_{i}\left(\sigma_{i_{1}}, \sigma_{i_{2}}, \ldots, \sigma_{i_{k}}\right),
$$

where $k$ is the number of regulatory inputs of $\sigma_{i}$. In the general case, and without applying any explicit knowledge about the connectivity of real gene networks or the combinations of values performed in real genes, we can assume those to be random. First, the presence of an interaction between gene $i$ and $j$ will depend on a certain probability, which is the definition of a random graph, yielding a Poisson distribution for the number $\langle k\rangle$ of incoming links. Second, to make functions random, for every combination of values in the inputs, the value of the output will be 1 with probability $p$ and 0 with probability $1-p$. This leaves a control over the bias in the function, but leaves it otherwise random. Summarizing, we have a discrete dynamics over a random graph with random functions of Boolean values, a Random Boolean Network (RBN).

Since Boolean networks are deterministic systems, whenever a network reaches a state which has already been visited in a previous time-step, it will enter in a cyclic trajectory. Due to this fact, the whole state space with $\Omega=2^{N}$ configurations is nicely partitioned into these cycles and their basins of attraction, i.e., the sets of states that lead to them. Basically, this defines a different graph in which nodes are the global states of the network, and the links represent the transitions between states due to the dynamics of the Boolean network. The basins are, in fact, simply the components of this graph. Since the dynamics gets trapped into the cycles of each basin once they are reached, basins can be also described in terms of the length of the cycles and also the length of the transition branches leading to them.

Kauffman identified these cycles with the cell types of an organism with a genome modeled by the Boolean network [Kauffman, 1993], since they represent disjoint possible stable states in the dynamics of a single network (given that all cells in an organism have the same genome). Varying the connectivity $K=\langle k\rangle$, he found profound differences in numbers, lengths and transition times of the emerging cycles. For high connectivity, $K \geq 5$, cycles and transients are very long, and there is a rather small number of basins. Also, perturbing a gene in the network 

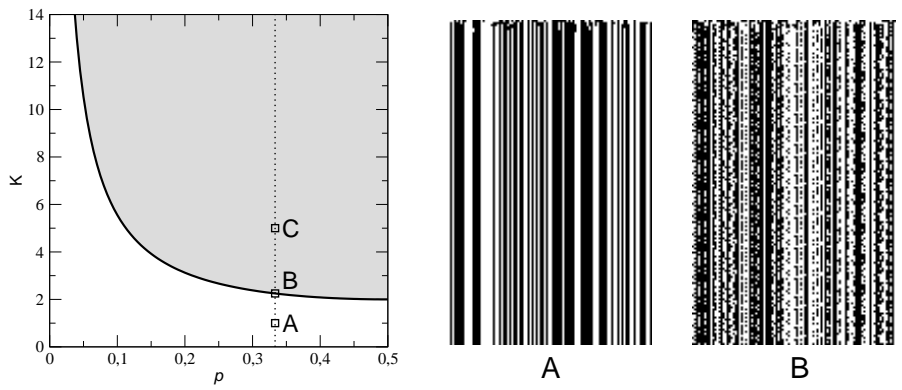

B

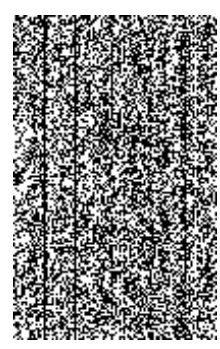

C

Figure 16: Left. Phase diagram of a random boolean network of parameters $K$ and $p$. The white area is the ordered regime, the grey area is the chaotic regime, and the critical phase is the black line in between. Right. Temporal dynamics of the genes in the network at the points $\mathrm{A}, \mathrm{B}$ and $\mathrm{C}$ along the line $p=1 / 3$ specified in the phase diagram. (A) Ordered, (B) Critical, and (C) Chaotic.

typically leads to another attractor. For low connectivity, $K=1$, cycles are usually fixed points, and transients are short, with again a high sensibility to perturbations. But for $K=2$, an unexpected order appears, what Kauffman called "order for free": the network has a small number of cycles and they are very robust against perturbations.

All this interesting properties about the cycles in the network can be explained by the existence of a phase transition [Aldana-González et al., 2003], that is, a sudden change in behavior for a smooth change in parameters. The only parameters in this model are $\langle k\rangle$ and $p$, which govern connectivity and the function bias, respectively. This allows us to draw a phase diagram, shown in figure 16 (left), which clearly separates the three distinct phases: ordered, chaotic, and critical. The critical phase is actually the interphase between the ordered and the chaotic one, whose threshold is defined by the equation

$$
K_{c}=\frac{1}{2 p(1-p)} .
$$

The names come from the observation of the dynamics in each case, which is also depicted in figure 16 (right). If we take a constant value of $p=1 / 3$ and just move the parameter $K$ (just as Kauffman did, although with $p=1 / 2$ ), we find that for low values of $K$, below 2.25 , the dynamics is frozen, and the values of gene expression stop switching altogether (fig. 16A), a behavior which resembles the description about the cycles. For higher values, above 2.25, we find that the dynamics is completely noisy and has no coherence (fig. 16C), which also would explain the cycle behavior. For exactly the value $K_{c}=2.25$, we can see that the dynamics is ordered and still not totally frozen, but with some complex periodicity (fig. 16B).

To approach this phase transition mathematically, we can perform a perturbative analysis, that is, we can ask what is the consequence of flipping the state of a small fraction of genes in the network. If these changes in value affect other 


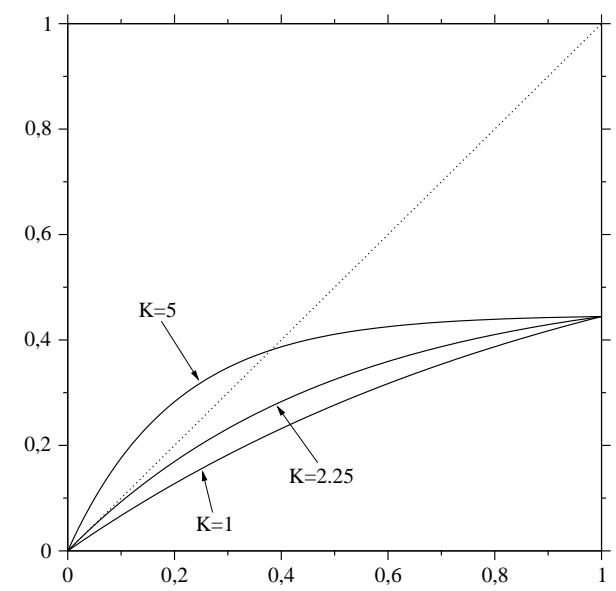

Figure 17: Graphical representation of $M(x)$ for different values of $K$. The values are the same as in figure 16 , showing chaotic phase $K=5$, critical phase $K_{c}=$ 2.25 , and ordered phase $K=1$. The circles show the fixed points of equation $x=M(x)$.

genes that have the damaged genes as inputs, these initial "errors" will propagate through the network, spreading the damage. Let us, then, consider the difference $\delta(t)$ of two configurations of values $\Sigma(t)=\left\{\sigma_{1}(t), \sigma_{2}(t), \ldots, \sigma_{N}(t)\right\}$ and $\Sigma^{\prime}(t)=\left\{\sigma_{1}^{\prime}(t), \sigma_{2}^{\prime}(t), \ldots, \sigma_{N}^{\prime}(t)\right\}$, which is defined as

$$
\delta(t)=\frac{1}{N} \sum_{i=1}^{N}\left|\sigma_{i}(t)-\sigma^{\prime}(t)\right| .
$$

If the temporal evolution of these two configurations is governed by the same Boolean network, with identical regulatory links and functions, the new configurations $\Sigma(t+1)$ and $\Sigma^{\prime}(t+1)$ will define a new value $\delta(t+1)$, which will indicate the tendency to grow or shrink of the differences. A tendency to shrink or grow would be identified as the ordered phase or chaotic phase, respectively, with the critical, maintaining phase in between, in which the damage neither expands nor shrinks on average.

To see what is the tendency of $\delta(t)$, we can look at the change in value of the units with inputs affected by changes, that is, if

$$
f_{i}\left(\sigma_{i_{1}}(t), \ldots, \sigma_{i_{k}}(t)\right)=f_{i}\left(\sigma_{i_{1}}^{\prime}(t), \ldots, \sigma_{i_{k}}^{\prime}(t)\right)
$$

with the probability that $\sigma_{k}(t) \neq \sigma_{k}^{\prime}(t)$ being $\delta(t)$. Since $f_{i}$ values are uncorrelated, any number of changes in the inputs of a gene will give a different, random value, and therefore the probability that the output will change is $2 p(1-p)$ (the probability that the $f(\Sigma(t))$ is different than $f\left(\Sigma^{\prime}(t)\right)$ ). If we use this for the dy- 

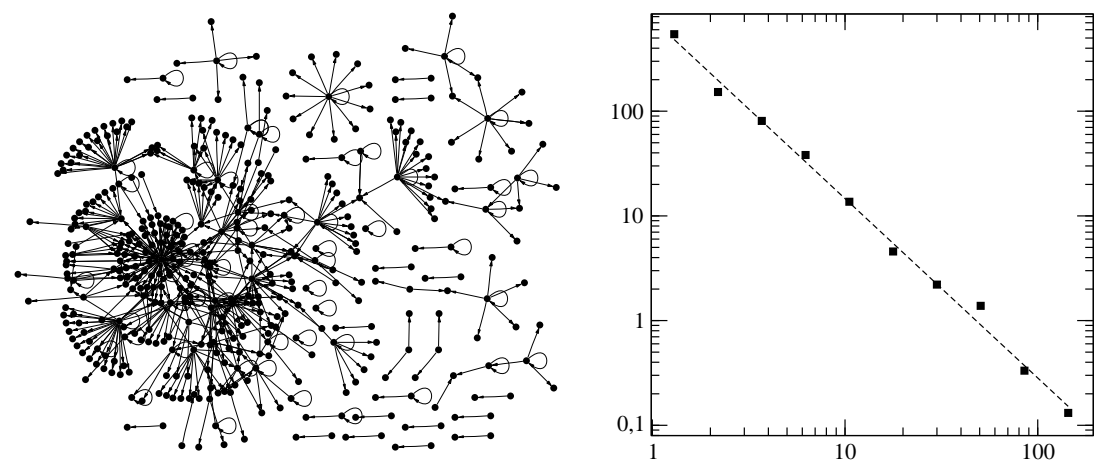

Figure 18: Left. Directed graph corresponding to a portion of the genetic regulatory network of E. coli. Right. Degree distribution of the genetic regulatory network of Yeast (from [Tong et al., 2004]).

namics of $\delta(t)$ we get

$$
\begin{aligned}
\delta(t+1) & =\sum_{k=1}^{\infty} 2 p(1-p)\left[1-[1-\delta(t)]^{k}\right] P_{\text {in }}(k) \\
& =2 p(1-p)\left[1-\sum_{k=1}^{\infty}[1-\delta(t)]^{k} P_{\text {in }}(k)\right]
\end{aligned}
$$

which is the same as saying that

$$
\delta(t+1)=M(\delta(t))
$$

with the mapping $M(x)$ being

$$
M(x) \equiv 2 p(1-p)\left[1-\sum_{k=1}^{\infty}(1-x)^{k} P_{\text {in }}(k)\right] .
$$

In the limit $t \rightarrow \infty$, the difference $\delta(t)$ will tend to the fixed point of equation $x=M(x)$. Even if $x=0$ is always a fixed point of eq. 29 , its stability depends on $P_{\text {in }}(k)$. Since $M$ is a monotonically increasing function of $x$, and also that $M(0)=0$ and $M(1)=2 p(1-p)$, eq. 29 will have a fixed point $x^{*} \neq 0$ only if $\lim _{x \rightarrow 0^{+}} M^{\prime}(x)>1$. In fact, this condition is the threshold for the ordered to chaotic transition. Figure 17 depicts the function $M(x)$ for different values of $p$ (the same as in figure 16), showing how $K_{c}=2.25$ is tangent to $f(x)=x$ at 0 .

From eq. 30 if follows that

$$
\lim _{x \rightarrow 0} \frac{d M(x)}{d x}=2 p(1-p) \sum_{k=1}^{\infty} k P_{\text {in }}(k)=1
$$

which is exactly the same as eq. 25 above. As we can see, the critical point for the dynamical transition in boolean netorks depends only on the average value of the 


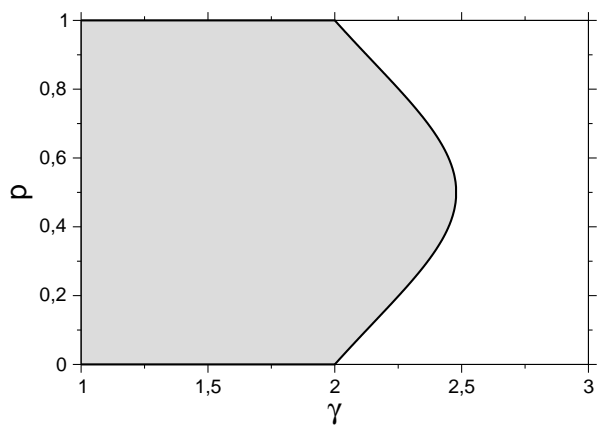

Figure 19: Phase diagram of the dynamical phase transition of a Boolean network with $P(k)=Z(\gamma) k^{-\gamma}$ as a function of $\gamma$ and $p$, to be compared with diagram in figure 16 (left). The grey area corresponds to the chaotic phase, and the white region to the ordered phase, with the black line being the critical phase.

distribution $P_{\text {in }}(k)$, and not on its exact shape. This result is useful, therefore, for any degree distribution, which is important since genetic regulatory networks are not really random.

Very recent data has confirmed that genetic regulatory networks are indeed scale-free [ref?]. Figure 18 shows a small graph corresponding to the regulatory network of $E$. coli (left) and the degree distribution for a the bigger network of $S$. cerevisiae (right). This new finding has stimulated research in Boolean networks which have a power-law degree distribution [Aldana, 2003, Aldana and Cluzel, 2003], further extending the results on random Boolean networks.

In a scale-free Boolean network, as mentioned, the condition of eq. 25 is still valid, and for a degree distribution $P(k)=Z(\gamma) k^{-\gamma}$, the average degree is determined by the expression

$$
\langle k\rangle=\sum_{k=0}^{\infty} k P(k)=\frac{Z(\gamma-1)}{Z(\gamma)},
$$

where $Z(\gamma)$ is the Riemann Zeta function, defined as $Z(\gamma)=\sum_{k=1}^{\infty} k^{-\gamma}$. Substituting this value in eq. 25 yields a transcendental equation involving $\gamma$. Using $\gamma$ as a descriptor of a power law distribution makes sense, since the variance in this kind of distributions is not bounded for $\gamma<3$, and hence the average degree is not a meaningful measure to characterize $P(k)$. The corresponding phase diagram using $\gamma$ (instead of $K$ ) and $p$ can be seen in figure 19 .

In the light of these results, is is conjectured that the extremely heterogeneous degree distribution of a scale-free network can provide a genome with two important properties at once [Aldana and Cluzel, 2003]. First, the robustness associated with biological systems, that the abundant ordered regime can provide. Second, due to the sensitivity of the network to changes of function or connectivity in highdegree nodes, the evolvability of the network is guaranteed. This stands in contrast to the insensitivity of a more homogeneous network, in which robustness necessarily makes adaptation very difficult. 


\section{Summary and discussion}

Exploring the static, graph-level structure of a complex system is allways limited by the many details that we miss but also by th elack of functionality. However, networks provide a pattern that results from a process of evolution and as such imposes severa constraints on possible explanatory mechanisms. On the other hand, the architecture of a network can reveal key features of the underlying functional organization. The modules found in protein contact maps were associated to well defined domains, but the details revelaed by the hierarchical clustering algorithm actually suggested that we are not facing a simple modular patter, but instead a hierarchical, nested architecture.

What can be learned about network origins from network structure? The standard view of evolution suggests that modules might have resulted from a process of selection in which the advantages introduced by specialized compartments would be enhanced by further selection. The models of proteome evolution suggests instead that once some thresholds of connectivity are reached, the patterns observed all emerge, including modularity.

Using networks as a theoretical framework seems to be relevant for a number of reasons. The first is that they provide well-defined quantitative properties to be reproduced by dynamical models of network evolution. Moreover, it is becoming clear that many network properties might be unique in terms of the universe of possible patterns of interaction among units in a complex network: once some minimal requirements such as a connected network with sparse connectivity are reached, other key features might emerge "for free". In such a view of network complexity, the architecture would be to a large extent a blueprint of underlying laws of organization. Dynamics, selection and adaptation would be shaped by such architectural constraints.

\section{Acknowledgements}

The authors would like to thank the members of the complex systems research group for useful discussions. This work was supported by a grant BFM2001-2154 (RVS), the Generalitat de Catalunya (PFD, 2001FI/00732) and The Santa Fe Institute.

\section{References}

B. Albert, A. Johnson, J. Lewis, M. Raff, K. Roberts, and P. Walter. Molecular Biology of the Cell. Garland Science, New York, 4th edition, 2002.

R. Albert and A.-L. Barabási. Statistical mechanics of complex networks. Rev. Mod. Phys., 74(47-97), 2002.

R. Albert and H. G. Othmer. The topology of the regulatory interactions predicts the expression pattern of the drosophila segment polarity genes. J. Theor. Biol., 223:1-18, 2003. 
M. Aldana. Boolean dynamics of networks with scale-free topology. Physica D, 185:45-66, 2003.

M. Aldana and P. Cluzel. A natural class of robust networks. PNAS, 100:87108714, 2003.

M. Aldana-González, S. Coppersmith, and L. P. Kadanoff. Boolean dynamics with random couplings. In E. Kaplan, J. E. Marsden, and K. R. Sreenivasan, editors, Perspectives and Problems in Nonlinear Science. A celebratory volume in honor of Lawrence Sirovich, Applied Mathematical Sciences. Springer, May 2003.

L. W. Ancel and W. Fontana. Plasticity, evolvability and modularity in rna. J. Exp. Zool., 288:242-283, 2000.

B. Bollobás. Random Graphs. Cambridge University Press, 2001. 2nd Edition.

S. N. Dorogovtsev and J. F. F. Mendes. Evolution of networks. From biological nets to Internet and $W W W$. Oxford University Press, Oxford, 2003.

A.M. Gutin, V.I. Abkevich, and E.I. Shakhnovich. Evolution-like selection of fastfolding model proteins. PNAS, 92(5):1282-1286, 1995.

T. Ito, T. Chiba, R. Ozawa, M. Yoshida, M. Hattori, and Y. Sakaki. A comprehensive two-hybrid analysis to explore the yeast protein interactome. PNAS, 98(8): 4569-4574, 2001.

S. A. Kauffman. The origins of order. Oxford U. Press, New York, 1993.

S.A. Kauffman. Metabolic stability and epigenesis in randomly constructed genetic nets. J.Theor.Biol., 22:437-467, 1969.

Li, S. et al. A Map of the Interactome Network of the Metazoan C. elegans. Science, 303(5657):540-543, 2004.

M. Maslov, K. Sneppen, and U. Alon. Correlation profiles and circuit motifs in complex networks. In S. Bornholdt and H.G. Schuster, editors, Handbook of Graphs and Networks, pages 168-198. John Wiley-VCH, 2002.

S. Maslov and K. Sneppen. Specificity and stability in topology of protein networks. Science, 296:910-913, 2002.

M. Newman. Random graphs as models of networks. In S. Bornholdt and H.G. Schuster, editors, Handbook of Graphs and Networks, pages 147-169. John Wiley-VCH, 2002a.

M. E. J. Newman. Assortative mixing in networks. Phys. Rev. Lett., 89:208701, 2002b.

E. Ravasz, A. L. Somera, D. A. Mongru, Z. N. Oltvai, and A.-L. Barabási. Hierarchical organization of modularity in metabolic networks. Science, 297:15511555, 2002. 
R. V. Solé, P. Fernández, and S. A. Kauffman. Adaptive walks in a gene network model of morphogenesis: insights into the cambrian explosion. IJDB, 47:685693, 2003.

R. V. Solé, R. Ferrer, J. M. Montoya, and S. Valverde. Selection, tinkering, and emergence in complex networks. Complexity, 8(1):20-33, 2002a.

R. V. Solé, R. Pastor-Satorras, E. Smith, and T. Kepler. A model of large-scale proteome evolution. Adv. Complex Systems, 5:43-54, 2002b.

R. V. Solé, I. Salazar-Ciudad, and S.A. Newman. Gene network dynamics and the evolution of development. Trends Ecol. Evol., 15:479-480, 2000.

N. M. Stadler, P. F. Stadler, G. P. Wagner, and W. Fontana. The topology of the possible: formal spaces underlying patterns of evolutionary change. J. Theor. Biol., 213:241-274, 2001.

Amy Hin Yan Tong, Guillaume Lesage, Gary D. Bader, Huiming Ding, Hong Xu, Xiaofeng Xin, James Young, Gabriel F. Berriz, Renee L. Brost, Michael Chang, YiQun Chen, Xin Cheng, Gordon Chua, Helena Friesen, Debra S. Goldberg, Jennifer Haynes, Christine Humphries, Grace He, Shamiza Hussein, Lizhu Ke, Nevan Krogan, Zhijian Li, Joshua N. Levinson, Hong Lu, Patrice Menard, Christella Munyana, Ainslie B. Parsons, Owen Ryan, Raffi Tonikian, Tania Roberts, Anne-Marie Sdicu, Jesse Shapiro, Bilal Sheikh, Bernhard Suter, Sharyl L. Wong, Lan V. Zhang, Hongwei Zhu, Christopher G. Burd, Sean Munro, Chris Sander, Jasper Rine, Jack Greenblatt, Matthias Peter, Anthony Bretscher, Graham Bell, Frederick P. Roth, Grant W. Brown, Brenda Andrews, Howard Bussey, and Charles Boone. Global mapping of the yeast genetic interaction network. Science, 303(5659):808-813, 2004.

G. von Dassow, E. Meir, E. Munro, and G. M. Odell. The segment polarity network is a robust developmental module. Nature, 406:188-192, 2000.

G. von Dassow and E. Munro. Modularity in animal development and evolution: elements of a conceptual framework for evodevo. J. Exp. Zool., 406(6792):188$192,1999$.

A. Vázquez. Growing network with local rules: Preferential attachment, clustering hierarchy, and degree correlations. Phys. Rev. E., 67(056104), 2003.

A. Vázquez, A. Flammini, A. Maritan, and A. Vespigniani. Modeling of protein interaction networks. ComPlexUs, 1:38-44, 2003.

G. P. Wagner. Homologues, natural kinds, and the evolution of modularity. Am. Zool., 36:36-43, 1996.

G. P. Wagner and L. Altenberg. Complex adaptations and the evolution of evolvability. Evolution, 50:967-976, 1996.

D. Watts and S. H. Strogatz. Collective dynamics of 'small-world' networks. $\mathrm{Na}$ ture, 393:440-442, 1998. 